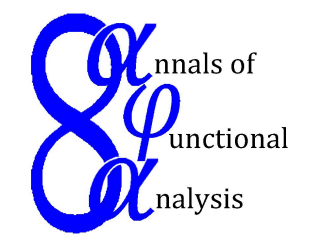

Ann. Funct. Anal. 6 (2015), no. 4, 226-246

http://doi.org/10.15352/afa/06-4-226

ISSN: 2008-8752 (electronic)

http://projecteuclid.org/afa

\title{
GEOMETRY AND OPERATOR THEORY ON QUATERNIONIC HILBERT SPACES
}

\author{
BINGZHE HOU ${ }^{1 *}$ AND GENG TIAN ${ }^{2}$ \\ Communicated by Y. Lim
}

\begin{abstract}
In this article, we study the geometry and operator theory on quaternionic Hilbert spaces. As it is well-known, Cowen-Douglas operators are a class of non-normal operators related to complex geometry on complex Hilbert spaces. Our purpose is to generalize this concept on quaternionic Hilbert spaces. At the beginning, we study a class of complex holomorphic curves which naturally induce complex vector bundles as sub-bundles in the product space of the base space and a quaternionic Hilbert space. Then we introduce quaternionic Cowen-Douglas operators and give their quaternion unitarily equivalent invariant related to the geometry of the holomorphic curves.
\end{abstract}

\section{INTRODUCTION}

Quaternions play an important role in quantum physics [1, 8]. From the mathematical point of view, it has resulted in spectral theorems for unitary and skewHermitian operators on quaternionic Hilbert spaces, and the study of unitary representations of groups in quaternionic Hilbert spaces [9, 11]. In the case of finite dimension, quaternionic matrices have been widely studied (see for example F. Zhang's survey [14]) and specially, the right eigenvalue problems were studied in $[5,6]$. In the case of infinite dimension, normal operators and unitary operators on quaternionic Hilbert spaces were well studied by K. Viswanath [13] and C. Sharma and T. Coulson [12].

On the other hand, since the beginning of the last century, mathematicians have been interested in creating a theory of quaternionic valued functions of one quaternionic variable, which would somehow resemble the classical theory of

Date: Received: Jan. 9, 2015; Accepted: Jun. 8, 2015.

* Corresponding author.

2010 Mathematics Subject Classification. Primary 46G20; Secondary 47B99, 51M15.

Key words and phrases. Cowen-Douglas operator, quaternionic Hilbert space, holomorphic curve, unitary equivalence. 
holomorphic functions of one complex variable. In recent years, F. Colombo et al. developed a new theory of regular functions of one quaternionic variable, which led to a functional calculus for quaternionic linear operators (see for example the monograph [3]).

In this article, our work relates to above two aspects. The basic idea is to introduce an analogue on quaternionic Hilbert space, of a class of non-normal operators given by Cowen and Douglas [4].

Definition 1.1. For $\Omega$ a connected open subset of $\mathbb{C}$ and $n$ a positive integer, let $\mathcal{B}_{n}(\Omega)$ denotes the operators $T$ in $\mathcal{L}(\mathcal{H})$ which satisfy:

(a) $\Omega \subseteq \sigma(T)=\{\omega \in \mathbb{C}: T-\omega$ not invertible $\}$;

(b) $\operatorname{Ran}(T-\omega)=\mathcal{H}$ for $\omega$ in $\Omega$;

(c) $\bigvee \operatorname{Ker}_{\omega \in \Omega}(T-\omega)=\mathcal{H}$; and

(d) $\operatorname{dim} \operatorname{Ker}(T-\omega)=n$ for $\omega$ in $\Omega$.

As it is well-known, the study of Cowen-Douglas operators are related to complex Hermitian geometry. In [4], Cowen and Douglas investigated that $\mathcal{B}_{n}(\Omega)$ correspond to holomorphic curves on complex Hilbert space, which naturally induce $n$-dimensional Hermitian holomorphic vector bundles, and proved that unitary classification of $\mathcal{B}_{n}(\Omega)$ is equivalent to the isomorphic classification of holomorphic curves. Furthermore, Jiang and Ji considered the similarity classification of holomorphic curves in [10].

Roughly speaking, Cowen-Douglas operators are a class of operators with "rich" point spectrum. We will generalize Cowen-Douglas operators on quaternionic Hilbert space as a class of quaternionic operators with "rich" right eigenvalues. The generalized operators correspond to a class of "holomorphic" curves. The definition of "holomorphy" means leaf-wise holomorphy on a holomorphic foliation of $\mathbb{H} \backslash \mathbb{R}$ in the spirit of Feres and Zeghib [7], which is different from slice regularity given by $\mathrm{F}$. Colombo et al.

In order to describe the generalization of Cowen-Douglas operators on quaternionic Hilbert space, we will investigate both geometry and operator theory on quaternionic Hilbert spaces. In section 2, some basic notations are given. We introduce a class of complex holomorphic curves in quaternionic Hilbert space in section 3, namely homogeneous leaf-wise holomorphic curves which naturally induce complex vector bundles as sub-bundles in the product space of the base space and a quaternionic Hilbert space. Then we give further discussions of their geometric properties in section 4 and section 5 . In particular, a rigidity theorem is obtained. In the last section, we describe an analogue of the class of Cowen-Douglas operators on quaternionic Hilbert spaces, which are related to the geometry of homogeneous leaf-wise holomorphic curves. Some examples are also exhibited.

\section{Preliminaries}

Let $\mathbb{H}$ be the skew field of real quaternions. Its elements are of the form of

$$
q=t_{0}+\mathbf{i} t_{1}+\mathbf{j} t_{2}+\mathbf{k} t_{3}, \quad t_{i} \in \mathbb{R}, i=0,1,2,3 .
$$


where

$$
\mathbf{i}^{2}=\mathbf{j}^{2}=\mathbf{k}^{2}=-1, \mathbf{i j}=-\mathbf{j i}=\mathbf{k}, \mathbf{j k}=-\mathbf{k j}=\mathbf{i} \text { and } \mathbf{k i}=-\mathbf{i k}=\mathbf{j} .
$$

$\mathbb{R}=\left\{q ; t_{1}=t_{2}=t_{3}=0\right\}$ will be identified with the real field and $\mathbb{C}=$ $\left\{q ; t_{2}=t_{3}=0\right\}$ with the complex field. $q^{*}=t_{0}-\mathbf{i} t_{1}-\mathbf{j} t_{2}-\mathbf{k} t_{3}$ will denote the conjugate of $q$ and $|q|=\sqrt{t_{0}^{2}+t_{1}^{2}+t_{2}^{2}+t_{3}^{2}}$ the absolute value of $q$. $\operatorname{Re}(q)=t_{0}$ and $\operatorname{Im}(q)=\mathbf{i} t_{1}+\mathbf{j} t_{2}+\mathbf{k} t_{3}$ are called the real part and imaginary part of $q$ respectively. Let

$$
\mathbb{S} \triangleq\left\{\mathbf{i} t_{1}+\mathbf{j} t_{2}+\mathbf{k} t_{3} ; t_{1}, t_{2}, t_{3} \in \mathbb{R} \text { and } t_{1}^{2}+t_{2}^{2}+t_{3}^{2}=1\right\}=\left\{x \in \mathbb{H} ; x^{2}=-1\right\} .
$$

$\mathbb{S}$ is said the imaginary unit sphere and the elements in it are said imaginary units. For any non-real quaternion $q$, define $I_{q}=\frac{\operatorname{Im}(q)}{|\operatorname{Im}(q)|} \in \mathbb{S}$. Thus,

Proposition 2.1. For any $q \in \mathbb{H} \backslash \mathbb{R}$, there exist, and are unique, $t_{0}, r \in \mathbb{R}$ with $r>0$, and $I_{q} \in \mathbb{S}$ such that $q=t_{0}+I_{q} r$.

For each $I \in \mathbb{S}$, denote $\mathbb{C}_{I} \triangleq\{x=t+r I ; t, r \in \mathbb{R}\}$ and $\mathbb{C}_{I}^{+} \triangleq\{x=t+r I ; t, r \in$ $\mathbb{R}$ and $r>0\}$. Notice that each $\mathbb{C}_{I}$ can be seemed as a complex plane. In the spirit of Feres and Zeghib [7], we consider a holomorphic foliation $\left\{\mathbb{C}_{I}^{+} ; I \in \mathbb{S}\right\}$ of $\mathbb{H} \backslash \mathbb{R}$, by which we mean that each of $\mathbb{C}_{I}^{+}, I \in \mathbb{S}$, carries the structure of a complex manifold and that this structure varies continuously on $\mathbb{H} \backslash \mathbb{R}$. Then we can define leaf-wise holomorphic functions on $\mathbb{H} \backslash \mathbb{R}$ respect to $\left\{\mathbb{C}_{I}^{+} ; I \in \mathbb{S}\right\}$. For $\Omega \subseteq \mathbb{H}$, denote $\Omega_{I}=\Omega \cap \mathbb{C}_{I}$ and $\Omega_{I}^{+}=\Omega \cap \mathbb{C}_{I}^{+}$.

For each $p, q \in \mathbb{H}$, if there exists an invertible element $x \in \mathbb{H}$ such that $x p=q x$, then $p$ and $q$ are said to be similar equivalent in $\mathbb{H}$. Moreover, if this $x$ is a qunitary, then $p$ and $q$ are said to be q-unitary equivalent in $\mathbb{H}$. Since $\mathbb{H}$ is a field, there is no difference between the two equivalence and we use ' $~ '$ to denote this equivalent relation. It is not difficult to see that, for $p, q \in \mathbb{H}, p$ and $q$ are equivalent in $\mathbb{H}$ if and only if $|p|=|q|$ and $\operatorname{Re}(p)=\operatorname{Re}(q)$. The concept of axially symmetric set was introduced in [2]. Now we give an equivalent definition of it.

Definition 2.2. A set $\Omega$ in $\mathbb{H}$ is said to be axially symmetric, if for any $\omega \in \Omega$, the equivalence class of $\omega$ contains in $\Omega$. For a set $\Omega$ in $\mathbb{H}$, the axially symmetric completion of $\Omega$ is the minimal axially symmetric set containing $\Omega$.

Let $U$ an open set of $\mathbb{C}$ and $z_{0} \in U$. A quaternion-valued function $f$ on $U$ is called right differentiable at $z_{0}$, if the limit $\lim _{\triangle z \rightarrow 0}\left(f\left(z_{0}+\triangle z\right)-f\left(z_{0}\right)\right)(\triangle z)^{-1}$ exists. In fact, we have some equivalent forms of this definition.

Proposition 2.3. Let $U$ an open set of $\mathbb{C}$ and $z_{0} \in U$. If $f$ is a quaternion-valued function on $U$, then the following statements are equivalent:

(1) $f$ is right differentiable at $z_{0}$;

(2) Write $z=x+\mathbf{i} y, x, y \in \mathbb{R}$ and $z_{0}=x_{0}+\left.\mathbf{i} y_{0} \cdot \frac{\partial f}{\partial x}\right|_{x=x_{0}}=\left.\frac{\partial f}{\partial y}\right|_{y=y_{0}} \cdot \mathbf{i}$;

(3) Write $f=g+\mathbf{j} h, g$ and $h$ are complex-valued functions on $U$. Both $g$ and $h$ are complex differentiable at $z_{0}$.

Notice that the quaternion-valued function $f$ is defined on a domain in $\mathbb{C}$ but not on a domain in $\mathbb{H}$. Thus the previous proposition is easy to obtain. Moreover, 
if $f$ is right differentiable at every point in open set $U$, we call that $f$ is right analytic on $U$. Consequently, we call that $f$ is right analytic at $z_{0}$ if $f$ is right analytic on a neighborhood of $z_{0}$.

Similarly, we call a quaternion-valued function $f$ defined on a domain in $\mathbb{C}$ is right coanalytic, left analytic or left coanalytic if the limits

or

$$
\begin{gathered}
\lim _{\triangle z \rightarrow 0}\left(f\left(z_{0}+\triangle z\right)-f\left(z_{0}\right)\right)(\overline{\triangle z})^{-1}, \\
\lim _{\triangle z \rightarrow 0}(\triangle z)^{-1}\left(f\left(z_{0}+\triangle z\right)-f\left(z_{0}\right)\right)
\end{gathered}
$$

$$
\lim _{\triangle z \rightarrow 0}(\overline{\triangle z})^{-1}\left(f\left(z_{0}+\triangle z\right)-f\left(z_{0}\right)\right)
$$

exist respectively. Replace $\mathbf{i}$ to $I$, we also can define them on $\mathbb{C}_{I}$. In the sequel, a holomorphic function on $\mathbb{C}_{I}$ (or $\mathbb{C}_{I}^{+}$) always means that it is right analytic on $\mathbb{C}_{I}$ (or $\mathbb{C}_{I}^{+}$). In addition, left coanalytic property will be also used in section 4 .

Definition 2.4. Let $\mathscr{V}$ be (right) a vector space over a field $\mathbb{K}$ ( which may be $\mathbb{R}, \mathbb{C}$ or $\mathbb{H}$ ). A quaternionic inner product on $\mathscr{V}$ is a map $<\cdot, \cdot>_{\mathbb{H}}: \mathscr{V} \times \mathscr{V} \rightarrow \mathbb{H}$ with the properties,

(i) $<x, y>_{\mathbb{H}}=\left(<y, x>_{\mathbb{H}}\right)^{*}$,

(ii) $<x p+y q, z>_{\mathbb{H}}=<x, z>_{\mathbb{H}} p+<y, z>_{\mathbb{H}} q$,

(ii') $<x, y p+z q>_{\mathbb{H}}=p^{*}<x, y>_{\mathbb{H}}+q^{*}<x, z>_{\mathbb{H}}$,

(iii) $\langle x, x\rangle_{\mathbb{H}} \geq 0$, and $\langle x, x\rangle_{\mathbb{H}}=0$ if and only if $x=0$,

for all $x, y, z \in \mathscr{V}$ and $p, q \in \mathbb{K}$.

Obviously, $\|x\|=\sqrt{\langle x, x\rangle_{\mathbb{H}}}$ is a norm on $\mathscr{V}$. Furthermore, a right vector space over the quaternions together with a quaternionic inner product on it which makes the resulting normed linear space complete is called a quaternionic Hilbert space.

Definition 2.5. Let $\mathcal{H}_{q}$ be a quaternionic Hilbert space. A subset $M$ in $\mathbb{H}$ is called a right linear manifold, if for any $x, y$ in $\mathcal{H}_{q}$ and any $p, q$ in $\mathbb{H}, x p+y q$ is also in $M$.

Definition 2.6. If $\mathcal{H}_{q}$ is a quaternionic Hilbert space, a linear operator $T: \mathcal{H}_{q} \rightarrow$ $\mathcal{H}_{q}$ is a function whose domain of definition is a right linear manifold, $\operatorname{dom} T$, in $\mathcal{H}_{q}$ and such that $T(x p+y q)=T(x) p+T(y) q$ for $x, y$ in $\operatorname{dom} T$ and $p, q$ in $\mathbb{H}$. $T$ is bounded if there is a constant $c>0$ such that $\|T x\| \leq c\|x\|$ for all $x$ in $\operatorname{dom} T$.

Throughout the rest of this article, we will denote by $\mathcal{H}_{q}$ a separable quaternionic Hilbert space and $\mathcal{H}_{c}$ a separable complex Hilbert space, $\mathcal{L}\left(\mathcal{H}_{q}\right)$ and $\mathcal{L}\left(\mathcal{H}_{c}\right)$ the set of all bounded (right) linear operators on $\mathcal{H}_{q}$ and $\mathcal{H}_{c}$ respectively. If fix an orthonormal basis (ONB in brief) $\left\{e_{n}\right\}_{n=1}^{\infty}$ in a quaternionic Hilbert space $\mathcal{H}_{q}$, then for $x \in \mathcal{H}_{q}$, we have $x=\sum_{n=1}^{\infty} e_{n} x_{n}$ and consequently write

$$
x=\left(x_{1}, x_{2}, \ldots\right)
$$

under this ONB, where $x_{n} \in \mathbb{H}$. For $T \in \mathcal{L}\left(\mathcal{H}_{q}\right)$, we can write

$$
T=\left[\begin{array}{ccc}
q_{11} & q_{12} & \ldots \\
q_{21} & q_{22} & \ldots \\
\vdots & \vdots & \ddots
\end{array}\right] \begin{gathered}
e_{1} \\
e_{2} \\
\vdots
\end{gathered}
$$


where $q_{i j} \in \mathbb{H}$. Moreover,

$$
T x=\left[\begin{array}{ccc}
q_{11} & q_{12} & \cdots \\
q_{21} & q_{22} & \ldots \\
\vdots & \vdots & \ddots
\end{array}\right]\left[\begin{array}{c}
x_{1} \\
x_{2} \\
\vdots
\end{array}\right]=\left[\begin{array}{c}
\sum_{j=1}^{\infty} q_{1 j} x_{j} \\
\sum_{j=1}^{\infty} q_{2 j} x_{j} \\
\vdots
\end{array}\right] .
$$

We also denote by $T^{*}$ the conjugate operator of $T$. In particular, an operator $U \in \mathcal{L}\left(\mathcal{H}_{q}\right)$ is quaternion unitary if $U^{*} U=U U^{*}=\mathcal{I}$, where $\mathcal{I}$ is the identity operator on $\mathcal{H}_{q}$. For $\omega \in \mathbb{H}$, we will use $T-\mathcal{I} \omega$ denote the operator on $\mathcal{H}_{q}$ defined by

$$
(T-\mathcal{I} \omega) x=T x-x \omega, \quad \text { for } x \in \mathcal{H}_{q} .
$$

Similarly, we define $T-\omega \mathcal{I}$ by

$$
(T-\omega \mathcal{I}) x=T x-\omega x, \quad \text { for } x \in \mathcal{H}_{q} .
$$

Notice that $T-\omega \mathcal{I}$ is right linear while $T-\mathcal{I} \omega$ is not right linear respect to quaternion field. However, $T-\mathcal{I} \omega$ will be considered in this paper since it is related to the right eigenvalues of quaternionic operators.

\section{A Class of COMPleX holomorphic CURVES in QUATERnionic Hilbert} SPACE

Let $\Omega$ be an axially symmetric domain in $\mathbb{H} \backslash \mathbb{R}$. In this section, we will introduce a class of sub-bundles in $\Omega \times \mathcal{H}_{q}$. In fact, they are complex vector bundles on $\Omega$, while it is also important of the manner of embedding in $\Omega \times \mathcal{H}_{q}$. To describe the manner of embedding, let us see some fundamental properties of quaternion field firstly.

For each $p, q \in \mathbb{H}$, denote $\mathscr{A}(p, q) \triangleq\{x \in \mathbb{H} ; x p=q x\}$. In particular, $\mathscr{A}(q, q)$ which means the commutant of $q$ in $\mathbb{H}$ is just $\mathbb{C}_{I_{q}}$. It is easy to see the following results.

Lemma 3.1. For any $I, J \in \mathbb{S}, I$ and $J$ are equivalent in $\mathbb{H}$. In fact, for any $I=\mathbf{i} t_{1}+\mathbf{j} t_{2}+\mathbf{k} t_{3} \in \mathbb{S}$, let

$$
\Lambda_{I}=\left\{\begin{array}{cl}
\mathbf{j}, & \text { if } I=-\mathbf{i} \\
\frac{\mathbf{i}\left(t_{1}+1\right)+\mathbf{j} t_{2}+\mathbf{k} t_{3}}{\sqrt{2+2 t_{1}}}, & \text { if } I \neq-\mathbf{i}
\end{array},\right.
$$

then $\Lambda_{I} \in \mathbb{S}$ and $\Lambda_{I} \mathbf{i}=I \Lambda_{I}$.

Lemma 3.2. Let $p, q \in \mathbb{H} \backslash \mathbb{R}$ and $0 \neq x \in \mathscr{A}(p, q)$. Then $\mathscr{A}(p, q)=\{x a ; a \in$ $\left.\mathbb{C}_{I_{p}}\right\}=\left\{b x ; b \in \mathbb{C}_{I_{q}}\right\}$.

Let $\mathbb{K}$ be a subfield of $\mathbb{H}$. For $n$ vectors $\left\{v_{i}\right\}_{i=1}^{n}$ in $\mathcal{H}_{q}$, denote $\bigvee_{\mathbb{K}}\left\{v_{i}, i=\right.$ $1, \ldots, n\}=\left\{v \in \mathcal{H}_{q} ; v=\sum_{i=1}^{n} v_{i} k_{i}, k_{i} \in \mathbb{K}\right\}$ and the vectors in $\bigvee_{\mathbb{K}}\left\{v_{i}, i=1, \ldots, n\right\}$ is said to be spanned by $\left\{v_{i}\right\}_{i=1}^{n}$ on $\mathbb{K}$. Moreover, for a infinite subset $A$ of $\mathcal{H}_{q}$, denote by $\bigvee_{\mathbb{K}} A$ the closure of all vectors finitely spanned by $A$ on $\mathbb{K}$. $\left\{v_{i}\right\}_{i=1}^{n}$ are called right linear independent on $\mathbb{K}$, if $\sum_{i=1}^{n} v_{i} k_{i}=0, k_{i} \in \mathbb{K}$ implies that every $k_{i}$ is zero. Furthermore, a $n$-dimensional $\mathbb{K}$-subspace $\mathscr{V}$ of $\mathcal{H}_{q}$ is a subset spanned by $n$ vectors $\left\{v_{i}\right\}_{i=1}^{n}$ which are right linear independent on $\mathbb{K}$, and denote $\operatorname{dim}_{\mathbb{K}} \mathscr{V}=n$. 
Denote by $\mathcal{G} r\left(n, \mathbb{K}, \mathcal{H}_{q}\right)$ the set of all $n$-dimensional $\mathbb{K}$-subspace of $\mathcal{H}_{q}$. If $\mathbb{K}=\mathbb{R}$, one can see $\mathcal{G} r\left(n, \mathbb{R}, \mathcal{H}_{q}\right)$ is a real Grassmann manifold. We are interested in the case $\mathbb{K}=\mathbb{C}_{I}$. Notice that for every $I \in \mathbb{S}, \mathcal{G} r\left(n, \mathbb{C}_{I}, \mathcal{H}_{q}\right)$ is a subset of $\mathcal{G} r\left(2 n, \mathbb{R}, \mathcal{H}_{q}\right)$.

Each $q \in \mathscr{A}(I, J) \backslash\{0\}$ induces a map $R_{q}: \mathcal{G} r\left(n, \mathbb{C}_{I}, \mathcal{H}_{q}\right) \rightarrow \mathcal{G} r\left(n, \mathbb{C}_{J}, \mathcal{H}_{q}\right)$ defined by

$$
R_{q}(\mathscr{V})=\left\{v q^{-1} ; v \in \mathscr{V}\right\} \text { for any } \mathscr{V} \in \mathcal{G} r\left(n, \mathbb{C}_{I}, \mathcal{H}_{q}\right)
$$

Proposition 3.3. Let $I, J \in \mathbb{S}$. For any $p, q \in \mathscr{A}(I, J) \backslash\{0\}, R_{p}=R_{q}$.

Proof. Suppose $p, q \in \mathscr{A}(I, J) \backslash\{0\}$. It follows from lemma 3.2 that there exits $\lambda \in \mathscr{A}(I, I) \backslash\{0\}$ such that $q=p \lambda$. Then for any $\mathscr{V} \in \mathcal{G} r\left(n, \mathbb{C}_{I}, \mathcal{H}_{q}\right)$,

$$
R_{q}(\mathscr{V})=\left\{v q^{-1} ; v \in \mathscr{V}\right\}=\left\{v \lambda^{-1} p^{-1} ; v \in \mathscr{V}\right\}=\left\{v p^{-1} ; v \in \mathscr{V}\right\}=R_{p}(\mathscr{V})
$$

Due to it, we denote the induced map by $R_{\mathscr{A}(I, J)}$.

Definition 3.4. Let $\Omega$ be a subset of $\mathbb{H} \backslash \mathbb{R}$. A map $f: \Omega \rightarrow \mathcal{G} r\left(2 n, \mathbb{R}, \mathcal{H}_{q}\right)$ is called a classical curve if $f(p) \in \mathcal{G} r\left(n, \mathbb{C}_{I_{p}}, \mathcal{H}_{q}\right)$ for each $p \in \Omega$.

Definition 3.5. Let $f$ be a classical curve on an axially symmetric subset $\Omega$ of $\mathbb{H} \backslash \mathbb{R}$. If for any $p, q \in \Omega$ with $p \sim q, R_{\mathscr{A}\left(I_{p}, I_{q}\right)} f(p)=f(q)$, then $f$ is said to be homogeneous.

Remark 3.6. Given any $I \in \mathbb{S}$. It follows from the definition that a homogeneous curve $f$ is determined by its restriction $\left.f\right|_{\Omega_{I}^{+}}$on $\Omega_{I}^{+}$. In addition, $\left.f\right|_{\Omega_{I}^{+}}$induces a complex vector bundle on $\Omega_{I}^{+}$as a subbundle in $\Omega_{I}^{+} \times \mathcal{H}_{q}$.

Definition 3.7. Let $\Lambda$ be a domain in $\mathbb{C}_{I}^{+}, I \in \mathbb{S}$. A function $g: \Lambda \rightarrow$ $\mathcal{G} r\left(n, \mathbb{C}_{I}, \mathcal{H}_{q}\right)$ is said to be holomorphic if there exist $n$ right analytic $\mathcal{H}_{q}$-valued functions $g_{1}(\lambda), \ldots, g_{n}(\lambda)$ on $\Lambda$ such that $g(\lambda)=\bigvee_{\mathbb{C}_{I}}\left\{g_{1}(\lambda), \ldots, g_{n}(\lambda)\right\}$ for each $\lambda \in \Lambda$. We call $\left\{g_{i}(\lambda)\right\}_{i=1}^{n}$ a frame of $g$.

Definition 3.8. Let $\Omega$ be a domain in $\mathbb{H} \backslash \mathbb{R}$. A classical curve

$$
f: \Omega \rightarrow \mathcal{G r}\left(2 n, \mathbb{R}, \mathcal{H}_{q}\right)
$$

is said to be leaf-wise holomorphic if, for every $I \in \mathbb{S}$, its restriction $\left.f\right|_{\Omega_{I}^{+}}: \Omega_{I}^{+} \rightarrow$ $\mathcal{G} r\left(n, \mathbb{C}_{I}, \mathcal{H}_{q}\right)$ is holomorphic. Moreover, the natural vector bundle $E_{f}$ (induced by $f$ ) on $\Omega$ is called a $n$-dimensional leaf-wise holomorphic complex vector bundle, that is,

$$
E_{f}=\left\{(v, \lambda) \in \mathcal{H}_{q} \times \Omega ; v \in f(\lambda)\right\} \text { and } \pi: E_{f} \rightarrow \Omega, \pi((v, \lambda))=\lambda .
$$

Given two leaf-wise holomorphic classical curves $f, \widetilde{f}: \Omega \rightarrow \mathcal{G} r\left(2 n, \mathbb{R}, \mathcal{H}_{q}\right)$. If there exists an quaternion unitary operator $U \in \mathcal{L}\left(\mathcal{H}_{q}\right)$ such that $\widetilde{f}=U f$, then $f$ and $\widetilde{f}$ are said to be congruent and $E_{f}$ and $E_{\widetilde{f}}$ are said to be equivalent.

At the end of this section, we give a similar conclusion to remark 3.6. In fact, it suffices to discuss the property of holomorphy. 
Proposition 3.9. Let $\Omega$ be an axially symmetric domain in $\mathbb{H} \backslash \mathbb{R}$ and let $f$ : $\Omega \rightarrow \mathcal{G} r\left(2 n, \mathbb{R}, \mathcal{H}_{q}\right)$ be a homogeneous curve. If $\left.f\right|_{\Omega_{I}^{+}}$is holomorphic for some $I \in \mathbb{S}$, then $f$ is leaf-wise holomorphic on $\Omega$.

Proof. According to definition 3.7, there exist $n$ right analytic $\mathcal{H}_{q}$-valued functions $f_{1}(\lambda), \ldots, f_{n}(\lambda)$ on $\Omega_{I}^{+}$such that $f(\lambda)=\bigvee_{\mathbb{C}_{I}}\left\{f_{1}(\lambda), \ldots, f_{n}(\lambda)\right\}$ for each $\lambda \in \Omega_{I}^{+}$. Let $f_{i}(\lambda)=\sum_{j=0}^{\infty} x_{j}^{(i)}\left(\lambda-\lambda_{0}\right)^{j}, i=1,2, \ldots, n, x_{j}^{(i)} \in \mathcal{H}_{q}$.

For any $J \in \mathbb{S}$, we will show that $\left.f\right|_{\Omega_{J}^{+}}: \Omega_{J}^{+} \rightarrow \mathcal{G} r\left(n, \mathbb{C}_{J}, \mathcal{H}_{q}\right)$ is holomorphic. Since $\Omega$ is an axially symmetric domain, for any $z \in \Omega_{J}^{+}$, there exist $p \in \mathcal{H}_{q} \backslash\{0\}$ and $\lambda \in \Omega_{I}^{+}$such that $z=p \lambda p^{-1}$. Define $g_{i}(z)=\sum_{j=0}^{\infty} x_{j}^{(i)} p^{-1}\left(z-z_{0}\right)^{j}$ for $i=1,2, \ldots, n$, where $z_{0}=p \lambda_{0} p^{-1}$

Since

$$
f_{i}(\lambda) p^{-1}=\sum_{j=0}^{\infty} x_{j}^{(i)}\left(\lambda-\lambda_{0}\right)^{j} p^{-1}=\sum_{j=0}^{\infty} x_{j}^{(i)} p^{-1}\left(z-z_{0}\right)^{j}=g_{i}(z),
$$

we have

$$
\bigvee_{\mathbb{C}_{J}}\left\{g_{1}(z), \ldots, g_{n}(z)\right\}=R_{p}(f(\lambda))=f(z) \text { for any } z \in \Omega_{J}^{+} .
$$

In addition, $\left\{g_{i}(z)\right\}_{i=1}^{n}$ obviously are right analytic $\mathcal{H}_{q}$-valued functions which implies that $\left.f\right|_{\Omega_{J}^{+}}: \Omega_{J}^{+} \rightarrow \mathcal{G} r\left(n, \mathbb{C}_{J}, \mathcal{H}_{q}\right)$ is holomorphic. Therefore, from definition $3.8, f$ is leaf-wise holomorphic on $\Omega$.

\section{Quaternionic extended Hermitian metrics on complex HOLOMORPHIC VECTOR BUNDLES}

Definition 4.1. Let $V_{1}$ and $V_{2}$ be two complex vector spaces. $\Phi: V_{1} \times V_{2} \rightarrow \mathbb{H}$ is called a quaternion-valued right conjugate bilinear functional if it satisfies the following conditions:

(1) $\Phi\left(\alpha \xi+\beta \xi^{\prime}, \eta\right)=\Phi(\xi, \eta) \alpha+\Phi\left(\xi^{\prime}, \eta\right) \beta$,

(2) $\Phi\left(\xi, \alpha \eta+\beta \eta^{\prime}\right)=\bar{\alpha} \Phi(\xi, \eta)+\bar{\beta} \Phi\left(\xi, \eta^{\prime}\right)$, by

where $\xi, \xi^{\prime} \in V_{1}, \eta, \eta^{\prime} \in V_{2}$ and $\alpha, \beta \in \mathbb{C}$. Moreover, define $\Phi^{*}: V_{2} \times V_{1} \rightarrow \mathbb{H}$

for any $\xi \in V_{1}$ and $\eta \in V_{2}$.

$$
\Phi^{*}(\eta, \xi)=(\Phi(\xi, \eta))^{*}
$$

Remark 4.2. One can write $\Phi=\varphi+\mathbf{j} \psi$, where $\varphi$ is a complex-valued conjugate bilinear functional from $V_{1} \times V_{2}$ to $\mathbb{C}$ and $\psi$ is a complex-valued bilinear functional from $V_{1} \times V_{2}$ to $\mathbb{C}$. $\varphi$ is called the complex part of $\Phi$ and $\psi$ is called the skew part of $\Phi$.

Definition 4.3. Let $(E, M, \pi)$ be a holomorphic vector bundle with rank $n$. For an open set $U \subseteq M$, a map $\widetilde{H}:\left.E\right|_{U} \times\left. E\right|_{U} \rightarrow \mathbb{H}$ is called a quaternionic extended Hermitian function on $U$ if it satisfies the following conditions,

(1) For any $(z, \omega) \in U \times U,\left.\widetilde{H}\right|_{E_{z} \times E_{\omega}}$ is a quaternion-valued right conjugate bilinear functional on $E_{z} \times E_{\omega}$;

(2) For any $(z, \omega) \in U \times U,\left.\widetilde{H}\right|_{E_{z} \times E_{\omega}}=\left(\left.\widetilde{H}\right|_{E_{\omega} \times E_{z}}\right)^{*}$; 
(3) $\widetilde{H}$ is right analytic respect to $z$ and left coanalytic respect to $\omega$.

Denote by $\mathscr{H}(U)$ all of the quaternionic extended Hermitian functions on $U$. Moreover, for any given point $x \in M$, denote by $\mathscr{H}_{x}$ the set of all quaternionic extended Hermitian functions on some open neighborhood of $x$.

Remark 4.4. The condition (2) means that

$$
\widetilde{H}(\xi, \eta)=(\widetilde{H}(\eta, \xi))^{*}, \quad \text { for any } \xi,\left.\eta \in E\right|_{U} .
$$

Similar to remark 4.2, each $\widetilde{H} \in \mathscr{H}(U)$ can be write in the form of $K+\mathbf{j} G$ where $K$ is called the complex part of $\widetilde{H}$ and $G$ is called the skew part of $\widetilde{H}$. Notice that both $K$ and $\mathbf{j} G$ are also elements in $\mathscr{H}(U)$. Moreover, the condition (3) holds if and only if $K$ is analytic respect to $z$ and coanalytic respect to $\omega$, and $G$ is analytic respect to both $z$ and $\omega$.

Now one can obtain an equivalent relation " $\sim_{x}$ " on $\mathscr{H}_{x}$. For $\widetilde{H}_{1}, \widetilde{H}_{2} \in \mathscr{H}_{x}$, $\widetilde{H}_{1} \sim_{x} \widetilde{H}_{2}$ if and only if there exists an open neighborhood $V$ of $x$ such that $\left.\widetilde{H}_{1}\right|_{E_{V} \times E_{V}}=\left.\widetilde{H}_{2}\right|_{E_{V} \times E_{V}}$. Denote by $\left[\widetilde{H}_{1}\right]_{x}$ the equivalence class of $\widetilde{H}_{1}$. Furthermore, we define $\mathscr{F}_{x}=\left\{[\widetilde{H}]_{x} ; \widetilde{H} \in \mathscr{H}_{x}\right\}$. Let $\mathscr{F}=\bigcup_{x \in M} \mathscr{F}_{x}$. For $U$ an open subset of $M$. Define

$$
\begin{aligned}
\mathscr{F}(U)= & \left\{s: U \rightarrow \mathscr{F} ; \text { for each } x \in U, s(x) \in \mathscr{F}_{x} \text { and there exist an open set } V\right. \\
& \text { with } \left.x \in V \subseteq U \text { and } \widetilde{H} \in \mathscr{H}(V) \text { such that }[\widetilde{H}]_{y}=s(y) \text { for any } y \in V\right\} .
\end{aligned}
$$

Then $\mathscr{F}$ is a sheaf, $\mathscr{F}_{x}$ is the stalk at $x$, and $\mathscr{F}(U)$ is the collection of all sections of $\mathscr{F}$ on $U$.

Notice that a section $s \in \mathscr{F}(U)$ may be not an element of $\mathscr{H}(U)$, while the local restrictions of $s$ are quaternionic extended Hermitian functions. To express this statement in precise, we firstly define that an open set $V \subseteq U$ is called representable for $s$ if there exists $\widetilde{H} \in \mathscr{H}(V)$ such that $[\widetilde{H}]_{x}=s(x)$ for every $x \in V$. Moreover, this $\widetilde{H}$ is called the representable function of $s$ on $V$. It follows from the definition of $\mathscr{F}(U)$ that for each $x \in U$, there exists an open neighborhood of $x$ which is representable for $s$.

Given a section $s \in \mathscr{F}(U)$. For every $x \in U$, write $s(x)=[\widetilde{H}]_{x}$. It follows from remark 4.4 that we have $\widetilde{H}=K+\mathbf{j} G$ where $K$ and $G$ are complex part and skew part of $\widetilde{H}$ respectively. Then define $s_{c}: U \rightarrow \mathscr{F}$ by $s_{c}(x)=[K]_{x}$, one can see $s_{c} \in \mathscr{F}(U) . s_{c}$ is called the complex part of section $s$. Similarly, $s_{s}(x) \triangleq[G]_{x}$ is called the skew part of section $s$.

For a section $s \in \mathscr{F}(U)$, write the complex part $s_{c}(x)=[K]_{x}$ and denote $\Delta_{U}$ the diagonal of $\left.E\right|_{U} \times\left. E\right|_{U}$, that is $\left\{E_{x} \times E_{x} ; x \in U\right.$. Define $H_{s}: \Delta_{U} \rightarrow \mathbb{C}$ by

$$
H_{s}(\xi, \eta)=H(\xi, \eta)
$$

for every $\xi, \eta \in \Delta_{U}$. We call $H_{s}$ the diagonal complex restriction of $s$ on $U$. It is not difficult to see that $H_{s}$ is a Hermitian structure on $\left.E\right|_{U}$ except for positivity by the conditions in definition 4.3 . 
Definition 4.5. A global section $s$ of $\mathscr{F}$ is called a quaternionic extended Hermitian (Kähler) metric (QEHM or QEKM in brief) on holomorphic vector bundle $(E, M, \pi)$, if the diagonal complex restriction $H_{s}$ of section $s$ is a Hermitian (Kähler) metric. Moreover, A holomorphic vector bundle with a QEHM (or QEKM) is briefly called QEHB (or QEKB).

To get a QEHM on a holomorphic vector bundle, the following result is useful which is easily obtained from the definition of QEHM.

Theorem 4.6. Let $(E, M, \pi)$ be a holomorphic vector bundle. A QEHM (QEKM) $s$ is determined by a family of pairs $\left(U_{\theta}, \widetilde{H}_{\theta}\right), \theta \in \Theta$, with the following properties.

(1) $\left\{U_{\theta}\right\}_{\theta \in \Theta}$ is an open cover of $M$.

(2) $\widetilde{H}_{\theta} \in \mathscr{H}(U)$ for $\theta \in \Theta$ and they are compatible, that means, if $U_{\theta} \cap U_{\tau} \neq \phi$ then $\widetilde{H}_{\theta}=\widetilde{H}_{\tau}$ on $\left.E\right|_{U_{\theta} \cap U_{\tau}} \times\left. E\right|_{U_{\theta} \cap U_{\tau}}$.

(3) For any $\theta \in \Theta$, the diagonal complex restriction $H_{\theta}$ of $\widetilde{H}_{\theta}$ is a Hermitian (or Kähler) metric on $U_{\theta}$.

In particular, if $M$ is representable for $s$, then $s$ is determined by only one function $\widetilde{H} \in \mathscr{H}(M)$. In this case, we say that $(E, M, \pi, s)$ is representable and $\widetilde{H}$ is the totally representable metric.

Let $(E, M, \pi)$ and $\left(E^{\prime}, M, \pi^{\prime}\right)$ be two holomorphic vector bundles and $\Psi: E^{\prime} \rightarrow$ $E$ a holomorphic isomorphism. Suppose that $s$ is a $\mathrm{QEHM}$ on $(E, M, \pi)$. Then $\Psi$ induces a QEHM $s_{\Psi}$ on $\left(E^{\prime}, M, \pi^{\prime}\right)$. According to theorem 4.6, choose a family of representable functions $\left(U_{\theta}, \widetilde{H}_{\theta}\right), \theta \in \Theta$. Define $\Psi_{*}\left(\widetilde{H}_{\theta}\right):\left.E^{\prime}\right|_{U_{\theta}} \times\left. E^{\prime}\right|_{U_{\theta}} \rightarrow \mathbb{H}$ by

$$
\Psi_{*}\left(\widetilde{H}_{\theta}\right)(\xi, \eta)=\widetilde{H}_{\theta}(\Psi(\xi), \Psi(\eta)),
$$

for any $\xi,\left.\eta \in E^{\prime}\right|_{U_{\theta}}$. Then $\left(U_{\theta}, \Psi_{*}\left(\widetilde{H}_{\theta}\right)\right)$ satisfy the properties in theorem 4.6 and consequently determine a QEHM $s_{\Psi}$. Notice that the QEHM $s_{\Psi}$ induced by $\Psi$ is independent on the choice of representable functions.

Definition 4.7. Let $(E, M, \pi, s)$ and $\left(E^{\prime}, M, \pi^{\prime}, s^{\prime}\right)$ be QEHBs and $\Psi: E^{\prime} \rightarrow E$ a holomorphic isomorphism. Then $\Psi$ is called a holomorphic q-isometry if $s^{\prime}=s_{\Psi}$.

Let $(E, M, \pi, H)$ be a Hermitian holomorphic vector bundle with Hermitian metric $H$ and $(E, M, \pi, s)$ a QEHB. If $H_{s}=H$, then $(E, M, \pi, s)$ is called a q-extension of $(E, M, \pi, H)$ and $(E, M, \pi, H)$ is called a complex restriction of $(E, M, \pi, s)$.

Proposition 4.8. Each $Q E H B(E, M, \pi, s)$ has a unique complex restriction.

The proof is simple.

Proposition 4.9. Each Hermitian holomorphic vector bundle $(E, M, \pi, H)$ has a q-extension.

Proof. For each trivialization $(U, \varphi), \varphi:\left.E\right|_{U} \rightarrow U \times \mathbb{C}^{n}$, there exists the Hermitian metric $H(z, \bar{z})$. Naturally, we can define $\widetilde{H}(z, w)=H(z, w)$ as required. 
Remark 4.10. Two q-extensions of a Hermitian holomorphic vector bundle $(E, M$, $\pi, H)$ may be not holomorphic q-isometric. More precisely, Let $(E, M, \pi, s)$ and $\left(E^{\prime}, M, \pi^{\prime}, s^{\prime}\right)$ be QEHBs. $\left(E, M, \pi, H_{s}\right)$ and $\left(E^{\prime}, M, \pi^{\prime}, H_{s^{\prime}}\right)$ being holomorphic isometric does not imply $(E, M, \pi, s)$ and $\left(E^{\prime}, M, \pi^{\prime}, s^{\prime}\right)$ being holomorphic qisometric. It is shown in the following example.

Example 4.11. Let $M=\{z \in \mathbb{C} ;|z-i|<1\}$ and let the two QEHBs $(M \times$ $\mathbb{C}, M, \pi, s),\left(M \times \mathbb{C}, M, \pi, s^{\prime}\right)$ define as follows: $s(x)=[\widetilde{H}]_{x}, s^{\prime}(x)=\left[\widetilde{H}^{\prime}\right]_{x}, x \in M$, where $\widetilde{H}:(M \times \mathbb{C}) \times(M \times \mathbb{C}) \rightarrow \mathbb{H}, \widetilde{H}((z, \alpha),(w, \beta))=\bar{\beta} \alpha$ and $\widetilde{H}^{\prime}:(M \times \mathbb{C}) \times$ $(M \times \mathbb{C}) \rightarrow \mathbb{H}, \widetilde{H}^{\prime}((z, \alpha),(w, \beta))=\bar{\beta} \alpha+\mathbf{j} \beta(z-w) \alpha$. Obviously, the complex restrictions of the two QEHBs are the same. However, the two QEHBs are not holomorphic q-isometric since for any complex holomorphic function $h$ on $M$

$$
\overline{h(w)}(\bar{\beta} \alpha) h(z) \neq \bar{\beta} \alpha+\mathbf{j} \beta(z-w) \alpha .
$$

\section{A RIGIDITY THEOREM: CONGRUENCE AND Q-ISOMETRY}

Let $\Omega$ be an axially symmetric domain in $\mathbb{H} \backslash \mathbb{R}$ and let $f: \Omega \rightarrow \mathcal{G} r\left(2 n, \mathbb{R}, \mathcal{H}_{q}\right)$ be a homogeneous leaf-wise holomorphic curve. Then $\left.f\right|_{\Omega_{\mathbf{i}}^{+}}: \Omega_{\mathbf{i}}^{+} \rightarrow \mathcal{G} r\left(n, \mathbb{C}, \mathcal{H}_{q}\right)$ induces a QEHB $\left(\left.E_{f}\right|_{\Omega_{\mathbf{i}}^{+}}, \Omega_{\mathbf{i}}^{+}, \pi, s\right)$, where the QEHM $s$ is determined by $\widetilde{H}$ : $\left.E_{f}\right|_{\Omega_{\mathrm{i}}^{+}} \times\left. E_{f}\right|_{\Omega_{\mathrm{i}}^{+}} \rightarrow \mathbb{H}$,

$$
\widetilde{H}(\xi, \eta)=<\xi, \eta>_{\mathbb{H}}
$$

for any $\xi \in f(z)$ and any $\eta \in f(w)$.

In addition, any frame $\left\{f_{1}, \ldots, f_{n}\right\}$ of $\left.f\right|_{\Omega_{\mathbf{i}}^{+}}$induces a QEHM $s_{f}$ on $\left(\Omega_{\mathbf{i}}^{+} \times\right.$ $\left.\mathbb{C}^{n}, \Omega_{\mathbf{i}}^{+}, \pi\right) . s_{f}$ is determined by $\widetilde{H}_{f}:\left(\Omega_{\mathbf{i}}^{+} \times \mathbb{C}^{n}\right) \times\left(\Omega_{\mathbf{i}}^{+} \times \mathbb{C}^{n}\right) \rightarrow \mathbb{H}$,

$$
\widetilde{H}_{f}(\alpha, \beta)=<\sum_{i=1}^{n} f_{i}(z) \alpha_{i}, \sum_{i=1}^{n} f_{i}(w) \beta_{i}>_{\mathbb{H}}
$$

for any $\alpha=\left(\alpha_{1}, \ldots, \alpha_{n}\right) \in\{z\} \times \mathbb{C}^{n}$ and $\beta=\left(\beta_{1}, \ldots, \beta_{n}\right) \in\{w\} \times \mathbb{C}^{n}$.

Proposition 5.1. Let $f: \Omega \rightarrow \mathcal{G} r\left(2 n, \mathbb{R}, \mathcal{H}_{q}\right)$ be a homogeneous leaf-wise holomorphic curve on $\Omega$ (an axially symmetric domain in $\mathbb{H} \backslash \mathbb{R}$ ) and let $\left\{f_{1}, \ldots, f_{n}\right\}$ be a frame of $\left.f\right|_{\Omega_{\mathbf{i}}^{+}}$. Then the two QEHBs $\left(\left.E_{f}\right|_{\Omega_{\mathbf{i}}^{+}}, \Omega_{\mathbf{i}}^{+}, \pi, s\right)$ and $\left(\Omega_{\mathbf{i}}^{+} \times \mathbb{C}^{n}, \Omega_{\mathbf{i}}^{+}, \pi, s_{f}\right)$ are holomorphic q-isometric.

Proof. Define $\Psi:\left.E_{f}\right|_{\Omega_{\mathbf{i}}^{+}} \rightarrow \Omega_{\mathbf{i}}^{+} \times \mathbb{C}^{n}$,

$$
\Psi\left(\sum_{i=1}^{n} f_{i}(z) \alpha_{i}\right)=\left(z,\left(\alpha_{1}, \ldots, \alpha_{n}\right)\right) .
$$

Then it follows from the construction of $s_{f}$ that $\Psi$ is a holomorphic q-isometry.

Moreover, in the sense of holomorphic q-isometry, the QEHBs $\left(\Omega_{\mathbf{i}}^{+} \times \mathbb{C}^{n}, \Omega_{\mathbf{i}}^{+}, \pi, s_{f}\right)$ induced by $f$ is independent on the choice of the frame of $\left.f\right|_{\Omega_{\mathbf{i}}^{+}}$. 
We denote by $\dot{+}$ the algebraic sum. For instance, if $f: \Omega \rightarrow \mathcal{G} r\left(2 n, \mathbb{R}, \mathcal{H}_{q}\right)$ is a curve, then

$$
\dot{+}_{p \in \Omega} f(p) \triangleq\left\{x ; x=\sum_{i=1}^{n} x_{i} \text { for any } n \in \mathbb{N}, \text { where } x_{i} \in f\left(p_{i}\right) \text { and } p_{i} \in \Omega\right\} .
$$

Lemma 5.2. Let $\Omega$ be an axially symmetric domain in $\mathbb{H} \backslash \mathbb{R}$ and let $f: \Omega \rightarrow$ $\mathcal{G} r\left(2 n, \mathbb{R}, \mathcal{H}_{q}\right)$ be a homogeneous leaf-wise holomorphic curve. Then

$$
\overline{\dot{+}_{p \in \Omega} f(p)}=\mathcal{H}_{q}
$$

if and only if

$$
\bigvee_{\mathbb{H}}\left\{f(\lambda) ; \lambda \in \Omega_{\mathbf{i}}^{+}\right\}=\mathcal{H}_{q}
$$

Proof. It is easily proved by the homogeneity of the curve $f$.

Furthermore, we can give equivalent descriptions of the condition $\overline{\dot{+}_{p \in \Omega} f(p)}=$ $\mathcal{H}_{q}$ locally.

Proposition 5.3. Suppose that $\Omega_{0}$ and $\Omega$ are two axially symmetric domain in $\mathbb{H} \backslash \mathbb{R}$ with $\Omega_{0} \subseteq \Omega$. Let $f: \Omega \rightarrow \mathcal{G} r\left(2 n, \mathbb{R}, \mathcal{H}_{q}\right)$ be a homogeneous leaf-wise holomorphic curve. Then

if and only if

$$
\overline{\dot{+}_{p \in \Omega} f(p)}=\mathcal{H}_{q}
$$

$$
\overline{\dot{+}_{p \in \Omega_{0}} f(p)}=\mathcal{H}_{q}
$$

Proof. Suppose $\overline{\dot{+}_{p \in \Omega} f(p)}=\mathcal{H}_{q}$. Let $\left\{f_{1}, \ldots, f_{n}\right\}$ be a frame of $\left.f\right|_{\Omega_{\mathrm{i}}^{+}}$. Consider $x \in \mathcal{H}_{q}$ which is orthogonal to $f(p)$ for $p \in \Omega_{0}$. Since the right analytic $\mathcal{H}_{q}$-valued functions $<f_{i}(z), x>_{\mathbb{H}}$ vanish on $\left(\Omega_{0}\right)_{\mathbf{i}}^{+}$and $\left(\Omega_{0}\right)_{\mathbf{i}}^{+}$is a nonempty open set in $\mathbb{C}^{+}$, they also vanish on $\Omega_{\mathbf{i}}^{+}$and hence $x=0$. Thus, $\overline{\dot{+}_{p \in \Omega_{0}} f(p)}=\overline{\dot{+}_{p \in \Omega} f(p)}=\mathcal{H}_{q}$. The contrary is obvious.

Proposition 5.4. Let $\Omega$ be an axially symmetric domain in $\mathbb{H} \backslash \mathbb{R}$ and let $f$ : $\Omega \rightarrow \mathcal{G} r\left(2 n, \mathbb{R}, \mathcal{H}_{q}\right)$ be a homogeneous leaf-wise holomorphic curve. Given a frame $\left\{f_{1}, \ldots, f_{n}\right\}$ of $\left.f\right|_{\Omega_{\mathbf{i}}^{+}}$and $\lambda_{0} \in \Omega_{\mathbf{i}}^{+}$. Then

$$
\overline{\dot{+}_{p \in \Omega} f(p)}=\mathcal{H}_{q}
$$

if and only if

$$
\bigvee_{\mathbb{H}}\left\{f_{i}^{(j)}\left(\lambda_{0}\right) ; i=1, \ldots, n \text { and } j=0,1, \ldots\right\}=\mathcal{H}_{q} .
$$

Proof. Since $f_{i}(z)$ are right analytic $\mathcal{H}_{q}$-valued functions, they have Taylor series with coefficients $f_{i}^{(j)}\left(\lambda_{0}\right)$ in some ball $\Omega_{0} \cap \mathbb{C}^{+}$of $\lambda_{0}$. Then together with lemma $5.2, \bigvee_{\mathbb{H}}\left\{f_{i}^{(j)}\left(\lambda_{0}\right) ; i=1, \ldots, n\right.$ and $\left.j=0,1, \ldots\right\}=\overline{\dot{+}_{p \in \Omega_{0}} f(p)}$. Therefore, we obtain the conclusion by proposition 5.3.

Similar to the rigidity theorem for complex holomorphic curves given by Cowen and Douglas in [4], we also get a rigidity theorem for homogeneous leaf-wise holomorphic curves. 
Theorem 5.5. (Rigidity) Let $\Omega$ be an axially symmetric domain in $\mathbb{H} \backslash \mathbb{R}$ and let $f, \widetilde{f}: \Omega \rightarrow \mathcal{G} r\left(2 n, \mathbb{R}, \mathcal{H}_{q}\right)$ be two homogeneous leaf-wise holomorphic curves such that $\overline{\dot{+}_{p \in \Omega} f(p)}=\overline{\dot{+}_{p \in \Omega} \widetilde{f}(p)}=\mathcal{H}_{q}$. Then $f$ and $\widetilde{f}$ are congruent if and only if the induced QEHBs $\left(\Omega_{\mathbf{i}}^{+} \times \mathbb{C}^{n}, \Omega_{\mathbf{i}}^{+}, \pi, s_{f}\right)$ and $\left(\Omega_{\mathbf{i}}^{+} \times \mathbb{C}^{n}, \Omega_{\mathbf{i}}^{+}, \pi, s_{\widetilde{f}}\right)$ are holomorphic q-isometric.

Proof. Suppose that $f$ and $\tilde{f}$ are congruent, i.e. there exists a quaternion unitary operator $U \in \mathcal{L}\left(\mathcal{H}_{q}\right)$ such that $U(f(\lambda))=\widetilde{f}(\lambda)$ for $\lambda \in \Omega$. It is easy to see that $U$ induces a holomorphic q-isometry between the two QEHBs $\left(\left.E_{f}\right|_{\Omega_{\mathbf{i}}^{+}}, \Omega_{\mathbf{i}}^{+}, \pi, s\right)$ and $\left(\left.E_{\widetilde{f}}\right|_{\Omega_{\mathbf{i}}^{+}}, \Omega_{\mathbf{i}}^{+}, \widetilde{\pi}, \widetilde{s}\right)$. In addition to proposition 5.1, the induced QEHBs $\left(\Omega_{\mathbf{i}}^{+} \times\right.$ $\left.\mathbb{C}^{n}, \Omega_{\mathbf{i}}^{+}, \pi, s_{f}\right)$ and $\left(\Omega_{\mathbf{i}}^{+} \times \mathbb{C}^{n}, \Omega_{\mathbf{i}}^{+}, \pi, s_{\widetilde{f}}\right)$ are holomorphic q-isometric.

Now suppose that the two induced QEHBs $\left(\Omega_{\mathbf{i}}^{+} \times \mathbb{C}^{n}, \Omega_{\mathbf{i}}^{+}, \pi, s_{f}\right)$ and $\left(\Omega_{\mathbf{i}}^{+} \times\right.$ $\left.\mathbb{C}^{n}, \Omega_{\mathbf{i}}^{+}, \pi, s_{\tilde{f}}\right)$ are holomorphic q-isometric, then $\left(\left.E_{f}\right|_{\Omega_{\mathbf{i}}^{+}}, \Omega_{\mathbf{i}}^{+}, \pi, s\right)$ and $\left(\left.E_{\widetilde{f}}\right|_{\Omega_{\mathbf{i}}^{+}}\right.$ $\left., \Omega_{\mathbf{i}}^{+}, \widetilde{\pi}, \widetilde{s}\right)$ are holomorphic q-isometric. Denote the q-isometric map by $\Phi$, the representable function of $s$ by $\widetilde{H}_{f}:\left.E_{f}\right|_{\Omega_{\mathbf{i}}^{+}} \times\left. E_{f}\right|_{\Omega_{\mathbf{i}}^{+}} \rightarrow \mathbb{H}$ and the representable function of $\widetilde{s}$ by $\widetilde{H}_{\widetilde{f}}:\left.E_{\widetilde{f}}\right|_{\Omega_{\mathbf{i}}^{+}} \times\left. E_{\widetilde{f}}\right|_{\Omega_{\mathbf{i}}^{+}} \rightarrow \mathbb{H}$. Since $\Phi$ is holomorphic q-isometric, we have

$$
<\xi, \eta>_{\mathbb{H}}=\widetilde{H}_{f}(\xi, \eta)=\widetilde{H}_{\widetilde{f}}(\Phi(\xi), \Phi(\eta))=<\Phi(\xi), \Phi(\eta)>_{\mathbb{H}}
$$

for any $\xi \in f(z)$ and any $\eta \in f(w)$, where $z, w \in \Omega_{\mathbf{i}}^{+}$.

Let $f_{i}(z), \tilde{f}_{i}(z), i=1, \ldots, n$ be right analytic $\mathcal{H}_{q^{\text {-valued }}}$ frames for $\left.f\right|_{\Omega_{\mathbf{i}}^{+}}$and $\left.\widetilde{f}\right|_{\Omega_{\mathrm{i}}^{+}}$respectively. We want to show

$$
<f_{i}^{(m)}(z), f_{j}^{(l)}(w)>_{\mathbb{H}}=<\widetilde{f}_{i}^{(m)}(z), \widetilde{f}_{j}^{(l)}(w)>_{\mathbb{H}}
$$

for $z, w \in \Omega_{\mathbf{i}}^{+}, i, j=1, \ldots, n$ and $m, l=0,1,2, \ldots$

Firstly, it is obvious for $m=l=0$.

Assume that the equation (5.1) holds for some $0 \leq m, l$. Then

$$
\begin{aligned}
& <f_{i}^{(m+1)}(z), f_{j}^{(l)}(w)>_{\mathbb{H}} \\
= & <\lim _{\Delta z \rightarrow 0}\left(f_{i}^{(m)}(z+\Delta z)-f_{i}^{(m)}(z)\right)(\Delta z)^{-1}, f_{j}^{(l)}(w)>_{\mathbb{H}} \\
= & \lim _{\Delta z \rightarrow 0}<\left(f_{i}^{(m)}(z+\Delta z)-f_{i}^{(m)}(z)\right)(\Delta z)^{-1}, f_{j}^{(l)}(w)>_{\mathbb{H}} \\
= & \lim _{\Delta z \rightarrow 0}<f_{i}^{(m)}(z+\Delta z)-f_{i}^{(m)}(z), f_{j}^{(l)}(w)>_{\mathbb{H}}(\Delta z)^{-1} \\
= & \lim _{\Delta z \rightarrow 0}<\widetilde{f}_{i}^{(m)}(z+\Delta z)-\widetilde{f}_{i}^{(m)}(z), \widetilde{f}_{j}^{(l)}(w)>_{\mathbb{H}}(\Delta z)^{-1} \\
= & \lim _{\Delta z \rightarrow 0}<\left(\widetilde{f}_{i}^{(m)}(z+\Delta z)-\widetilde{f}_{i}^{(m)}(z)\right)(\Delta z)^{-1}, \widetilde{f}_{j}^{(l)}(w)>_{\mathbb{H}} \\
= & <\lim _{\Delta z \rightarrow 0}\left(\widetilde{f}_{i}^{(m)}(z+\Delta z)-\widetilde{f}_{i}^{(m)}(z)\right)(\Delta z)^{-1}, \widetilde{f}_{j}^{(l)}(w)>_{\mathbb{H}} \\
= & <\widetilde{f}_{i}^{(m+1)}(z), \widetilde{f}_{j}^{(l)}(w)>_{\mathbb{H}} .
\end{aligned}
$$

Similarly, we have

$$
<f_{i}^{(m)}(z), f_{j}^{(l+1)}(w)>_{\mathbb{H}}=<\widetilde{f}_{i}^{(m)}(z), \widetilde{f}_{j}^{(l+1)}(w)>_{\mathbb{H}} .
$$


Therefore, by induction principle, we obtain

$$
<f_{i}^{(m)}(z), f_{j}^{(l)}(w)>_{\mathbb{H}}=<\widetilde{f}_{i}^{(m)}(z), \widetilde{f}_{j}^{(l)}(w)>_{\mathbb{H}}
$$

for $z, w \in \Omega_{\mathbf{i}}^{+}, i, j=1, \ldots, n$ and $m, l=0,1,2, \ldots$.

Next, we will define the quaternion unitary operator as required. According to proposition 5.4, one can see

$$
\bigvee_{\mathbb{H}}\left\{f_{i}^{(m)}(z) ; i=1, \ldots, n \text { and } m=0,1, \ldots\right\}=\mathcal{H}_{q}
$$

and

$$
\bigvee_{\mathbb{H}}\left\{\widetilde{f}_{i}^{(m)}(z) ; i=1, \ldots, n \text { and } m=0,1, \ldots\right\}=\mathcal{H}_{q} .
$$

Thus we can define $U_{z}$ from $\mathcal{H}_{q}$ to $\mathcal{H}_{q}$ by

$$
U_{z} f_{i}^{(m)}(z)=\widetilde{f}_{i}^{(m)}(z),
$$

for $1 \leq i \leq n$ and all $m \in \mathbb{N}$. Moreover, $U_{z}$ is isometric, since equation (5.1) implies

$$
<U_{z} f_{i}^{(m)}(z), U_{z} f_{j}^{(l)}(z)>_{\mathbb{H}}=<\widetilde{f}_{i}^{(m)}(z), \widetilde{f}_{j}^{(l)}(z)>_{\mathbb{H}}
$$

for $1 \leq i, j \leq n$ and all $m, l \in \mathbb{N}$, and thus is well-defined. Therefore, $U_{z}$ is a quaternion unitary operator on $\mathcal{H}_{q}$. We should also show that $U_{z}$ is independent on the choice of $z$. Given any $z_{0} \in \Omega_{\mathbf{i}}^{+}$. If $z$ belongs to a small open neighborhood of $z_{0}$, then

$$
f_{i}^{(m)}(z)=\sum_{j=0}^{\infty} f_{i}^{(m+j)}\left(z_{0}\right) \frac{\left(z-z_{0}\right)^{j}}{j !} .
$$

Since $U_{z_{0}}$ is bounded, we have

$$
\begin{aligned}
U_{z_{0}}\left(f_{i}^{(m)}(z)\right) & =\sum_{j=0}^{\infty} U_{z_{0}}\left(f_{i}^{(m+j)}\left(z_{0}\right)\right) \frac{\left(z-z_{0}\right)^{j}}{j !} \\
& =\sum_{j=0}^{\infty} \widetilde{f}_{i}^{(m+j)}\left(z_{0}\right) \frac{\left(z-z_{0}\right)^{j}}{j !} \\
& =\widetilde{f}_{i}^{(m)}(z) \\
& =U_{z}\left(f_{i}^{(m)}(z)\right)
\end{aligned}
$$

hence $U_{z}=U_{z_{0}}$ and $U_{z}$ is independent of $z \in \Omega_{\mathbf{i}}^{+}$. If we set $U=U_{z}$, then $U f(z)=\widetilde{f}(z)$ for $z \in \Omega_{\mathbf{i}}^{+}$. By the homogeneity of $f$ and $\widetilde{f}$, we obtain they are congruent.

\section{Analogues of Cowen-Douglas operators on quaternionic HILBERT SPACES}

Firstly, let us recall the right eigenvalues of $T \in \mathcal{L}\left(\mathcal{H}_{q}\right)$ defined by

$$
\Sigma_{r}(T)=\left\{\omega \in \mathbb{H} ; \text { there exists nontrivial } x \in \mathcal{H}_{q} \text { such that } T x=x \omega .\right\}
$$

Now, it is time to introduce the definition of quaternionic Cowen-Douglas operators. 
Definition 6.1. For $\Omega$ a connected open subset of $\mathbb{H} \backslash \mathbb{R}$ and $n$ a positive integer, let $\mathcal{R} \mathcal{Q} \mathcal{B}_{n}(\Omega)$ denote the operators $T$ in $\mathcal{L}\left(\mathcal{H}_{q}\right)$ which satisfy:

(a) $\Omega \subseteq \Sigma_{r}(T)$;

(b) $\operatorname{Ran}(T-\mathcal{I} \omega) \triangleq\left\{T x-x \omega \mid x \in \mathcal{H}_{q}\right\}=\mathcal{H}_{q}$ for $\omega$ in $\Omega$;

(c) $\overline{\dot{+}_{\omega \in \Omega} \operatorname{Ker}(T-\mathcal{I} \omega)}=\mathcal{H}_{q}$, where $\operatorname{Ker}(T-\mathcal{I} \omega) \triangleq\left\{x \in \mathcal{H}_{q} \mid T x=x \omega\right\}$;

(d) For $\omega$ in $\Omega, \operatorname{dim}_{\mathbb{C}_{I \omega}}(\operatorname{Ker}(T-\mathcal{I} \omega))=n$. $n$ is called the index of $T$ at $\omega$ (or on $\Omega$ ).

We call the operators in $\mathcal{R Q}_{\mathcal{Q}} \mathcal{B}_{n}(\Omega)$ right Cowen-Douglas operators on quaternionic Hilbert spaces or briefly right quaternionic Cowen-Douglas operators.

Remark 6.2. Let $T \in \mathcal{R} \mathcal{Q B}_{n}(\Omega)$. For any $\omega$ in $\Omega, \operatorname{Ker}(T-\mathcal{I} \omega)$ is an $n$-dimensional $\mathbb{C}_{I_{\omega}}$-subspace of $\mathcal{H}_{q}$, i.e., for any $x \in \operatorname{Ker}(T-\mathcal{I} \omega)$ and $q \in \mathbb{C}_{I_{\omega}}$,

$$
T(x q)=(T x) q=(x \omega) q=x(\omega q)=x(q \omega)=(x q) \omega,
$$

which implies $x q \in \operatorname{Ker}(T-\mathcal{I} \omega)$. In fact, it is isomorphic to an $n$-dimensional complex vector space since $\mathbb{C}_{I_{\omega}}$ is naturally isomorphic to $\mathbb{C}$.

Lemma 6.3. Let $T \in \mathcal{L}\left(\mathcal{H}_{q}\right)$ and $\omega \in \Sigma_{r}(T)$. Then for any $q \in \mathbb{H} \backslash\{0\}$, we have $q \omega q^{-1} \in \Sigma_{r}(T)$ and $\{\operatorname{Ker}(T-\mathcal{I} \omega)\} q^{-1}=\operatorname{Ker}\left(T-\mathcal{I} q \omega q^{-1}\right)$.

Proof. Suppose $x \in \operatorname{Ker}(T-\mathcal{I} \omega)$.Then

$$
T\left(x q^{-1}\right)=x \omega q^{-1}=\left(x q^{-1}\right)\left(q \omega q^{-1}\right) .
$$

Proposition 6.4. Let $\Omega$ be a connected open subset of $\mathbb{H} \backslash \mathbb{R}$ and $T \in \mathcal{R} \mathcal{Q} \mathcal{B}_{n}(\Omega)$. If $\widetilde{\Omega}$ is the axially symmetric completion of $\Omega$, then $T \in \mathcal{R} \mathcal{Q} \mathcal{B}_{n}(\widetilde{\Omega})$.

Proof. We will show that $T$ satisfies conditions $(a-d)$ in definition 6.1.

a) It follows from lemma 6.3 that $\widetilde{\Omega} \subseteq \Sigma_{r}(T)$.

b) For any $p \in \widetilde{\Omega}$, there exist $\omega \in \Omega$ and $q \in \mathbb{H} \backslash\{0\}$ such that $p=q \omega q^{-1}$. For any $y \in \mathcal{H}_{q}$, it follows from $\operatorname{Ran}(T-\mathcal{I} \omega)=\mathcal{H}_{q}$ that there exists $x \in \mathcal{H}_{q}$ such that $T x-x \omega=y q$. Then $(T x) q^{-1}-(x \omega) q^{-1}=y$, that is $T\left(x q^{-1}\right)-\left(x q^{-1}\right)\left(q \omega q^{-1}\right)=y$. Hence $y \in \operatorname{Ran}(T-\mathcal{I} p)$.

c) $\overline{\dot{+}_{\omega \in \Omega} \operatorname{Ker}(T-\mathcal{I} \omega)}=\mathcal{H}_{q}$ obviously implies $\overline{\dot{+}_{\omega \in \widetilde{\Omega}} \operatorname{Ker}(T-\mathcal{I} \omega)}=\mathcal{H}_{q}$ since $\Omega \subseteq \widetilde{\Omega}$.

d) For any $q \in \mathbb{H} \backslash\{0\}$ and $\omega \in \Omega$, since $\{\operatorname{Ker}(T-\mathcal{I} \omega)\} q^{-1}=\operatorname{Ker}\left(T-\mathcal{I} q \omega q^{-1}\right)$, we have

$$
\operatorname{dim}_{\mathbb{C}_{I_{q \omega q}-1}}\left(\operatorname{Ker}\left(T-\mathcal{I} q \omega q^{-1}\right)\right)=n
$$

Remark 6.5. The condition $(c)$ in definition 6.1 is equivalent to $\bigvee_{\mathbb{H}}\{\operatorname{Ker}(T-$ $\left.\mathcal{I} \omega) ; \omega \in \Omega_{\mathbf{i}}^{+}\right\}=\mathcal{H}_{q}$, and the condition $(d)$ in definition 6.1 is equivalent to

$$
\operatorname{dim}_{\mathbb{C}} \operatorname{Ker}\left(T_{c}-\omega\right)=n \quad \text { for } \omega \in \Omega_{\mathbf{i}}^{+} .
$$

In the research of quaternionic linear operators, complex representation is a useful technology. Let $\left\{e_{i}\right\}_{i=1}^{\infty}$ be an ONB of $\mathcal{H}_{q}$. Denote $\mathcal{H}_{1}=\bigvee_{\mathbb{C}}\left\{e_{n} ; n=\right.$ $1,2, \ldots\}$ and $\mathcal{H}_{2}=\bigvee_{\mathbb{C}}\left\{e_{n} \mathbf{j} ; n=1,2, \ldots\right\}$. Then $\mathcal{H}_{1} \oplus \mathcal{H}_{2}$ is a complex Hilbert 
space. For every $x \in \mathcal{H}_{q}$, we can write uniquely $x=u+\mathbf{j} v$ where $u \in \mathcal{H}_{1}$ and $v \in \mathcal{H}_{2}$. Then the complex representation of $x$ is $x_{c}=(u, v)$ in $\mathcal{H}_{1} \oplus \mathcal{H}_{2}$. Moreover, for every quaternionic linear operator $T$ on $\mathcal{H}_{q}$, we can give a complex representation of $T$ defined by $T_{c}: \mathcal{H}_{1} \oplus \mathcal{H}_{2} \rightarrow \mathcal{H}_{1} \oplus \mathcal{H}_{2}$

$$
T_{c}=\left[\begin{array}{cc}
T_{1} & -\overline{T_{2}} \\
T_{2} & \overline{T_{1}}
\end{array}\right] \begin{aligned}
& \mathcal{H}_{1} \\
& \mathcal{H}_{2}
\end{aligned}
$$

where $T_{1}$ and $T_{2}$ are complex linear operators determined by $T$. If a linear operator is seemed as an infinite dimensional matrix, we can write $T=T_{1}+\mathbf{j} T_{2}$. Computing directly, one can get the following result.

Lemma 6.6. Let $T, S$ be two quaternionic linear operators on $\mathcal{H}_{q}$ and $x \in \mathcal{H}_{q}$. Then

$$
(T+S)_{c}=T_{c}+S_{c}, \quad(T S)_{c}=T_{c} S_{c} \text { and }(T x)_{c}=T_{c} x_{c} .
$$

Furthermore, for any $\lambda \in \mathbb{C}$, we have

$$
(T-\mathcal{I} \lambda)_{c}=T_{c}-\lambda .
$$

According to complex representation and the equation (6.1), one can see

Theorem 6.7. $T \in \mathcal{R} \mathcal{Q} \mathcal{B}_{n}(\Omega)$ if and only if $T_{c}$ satisfies the following conditions.

(1) $\Omega_{\mathbf{i}}^{+} \subseteq \sigma_{p}\left(T_{c}\right)$, where $\sigma_{p}\left(T_{c}\right)$ means the point spectrum of $T_{c}$;

(2) $\operatorname{Ran}\left(T_{c}-\omega\right)=\mathcal{H}_{1} \oplus \mathcal{H}_{2}$ for $\omega \in \Omega_{\mathbf{i}}^{+}$;

(3) $\bigvee_{\mathbb{C}}\left\{\operatorname{Ker}\left(T_{c}-\omega\right), \operatorname{Ker}\left(T_{c}-\bar{\omega}\right) ; \omega \in \Omega_{\mathbf{i}}^{+}\right\}=\mathcal{H}_{1} \oplus \mathcal{H}_{2}$;

(4) $\operatorname{dim}_{\mathbb{C}} \operatorname{Ker}\left(T_{c}-\omega\right)=n$ for $\omega \in \Omega_{\mathbf{i}}^{+}$.

Proof. Obviously, the condition (1) is equivalent to the condition (a) in definition 6.1 and (2) is equivalent to (b). By remark 6.5, the condition (4) is equivalent to the condition $(\mathrm{d})$ in definition 6.1. Now prove the condition (3) is equivalent to the condition (c) in definition 6.1. In fact, we need only to prove

$$
\left(\bigvee_{\mathbb{H}}\left\{\operatorname{Ker}(T-\mathcal{I} \omega) ; \omega \in \Omega_{\mathbf{i}}^{+}\right\}\right)_{c}=\bigvee_{\mathbb{C}}\left\{\operatorname{Ker}\left(T_{c}-\omega\right), \operatorname{Ker}\left(T_{c}-\bar{\omega}\right) ; \omega \in \Omega_{\mathbf{i}}^{+}\right\} .
$$

Notice that for any $\lambda \in \Omega_{\mathbf{i}}, x \in \operatorname{Ker}(T-\lambda)$ if and only if $x_{c} \in \operatorname{Ker}\left(T_{c}-\lambda\right)$. Given any $\omega \in \Omega_{\mathbf{i}}^{+}$. For any $x \in \operatorname{Ker}(T-\mathcal{I} \omega)$ and any $q=\alpha+\mathbf{j} \beta \in \mathbb{H}, x q=x \alpha+(x \mathbf{j}) \beta$. Since $\omega \in \Omega_{\mathbf{i}}^{+}, \mathbf{j}^{-1} \omega \mathbf{j}=\bar{\omega}$ and consequently $x \mathbf{j} \in \operatorname{Ker}(T-\mathcal{I} \bar{\omega})$. Then

$$
(x q)_{c}=x_{c} \alpha+(x \mathbf{j})_{c} \beta, \quad x_{c} \alpha \in \operatorname{Ker}\left(T_{c}-\omega\right) \text { and }(x \mathbf{j})_{c} \beta \in \operatorname{Ker}\left(T_{c}-\bar{\omega}\right),
$$

and hence

$$
\left(\bigvee_{\mathbb{H}}\left\{\operatorname{Ker}(T-\mathcal{I} \omega) ; \omega \in \Omega_{\mathbf{i}}^{+}\right\}\right)_{c} \subseteq \bigvee_{\mathbb{C}}\left\{\operatorname{Ker}\left(T_{c}-\omega\right), \operatorname{Ker}\left(T_{c}-\bar{\omega}\right) ; \omega \in \Omega_{\mathbf{i}}^{+}\right\}
$$

On the other hand, for any $u \in \operatorname{Ker}\left(T_{c}-\omega\right)$ and $v \in \operatorname{Ker}\left(T_{c}-\bar{\omega}\right)$, there exist $x, y \in \operatorname{Ker}(T-\mathcal{I} \omega)$ such that $x_{c}=u$ and $(y \mathbf{j})_{c}=v$. Thus

$$
\left(\bigvee_{\mathbb{H}}\left\{\operatorname{Ker}(T-\mathcal{I} \omega) ; \omega \in \Omega_{\mathbf{i}}^{+}\right\}\right)_{c} \supseteq \bigvee_{\mathbb{C}}\left\{\operatorname{Ker}\left(T_{c}-\omega\right), \operatorname{Ker}\left(T_{c}-\bar{\omega}\right) ; \omega \in \Omega_{\mathbf{i}}^{+}\right\}
$$

Next, it is investigated that quaternionic Cowen-Douglas operators are really a class of geometric operators. 
Theorem 6.8. For every $n \in \mathbb{N}$, each operator $T$ in $\mathcal{R} \mathcal{Q} \mathcal{B}_{n}(\Omega)$ naturally induces an $n$-dimensional homogeneous leaf-wise holomorphic curve $f_{T}$ on $\Omega$.

Proof. Define $f_{T}: \Omega \rightarrow \mathcal{G r}\left(2 n, \mathbb{R}, \mathcal{H}_{q}\right)$ by

$$
f_{T}(\omega)=\operatorname{Ker}(T-\mathcal{I} \omega)
$$

It follows from the proof (d) of proposition 6.4 that $f_{T}$ is a homogeneous curve. According to proposition 3.9, it suffices to show that $\left.f_{T}\right|_{\Omega_{\mathbf{i}}^{+}}: \Omega_{\mathbf{i}}^{+} \rightarrow \mathcal{G} r\left(n, \mathbb{C}, \mathcal{H}_{q}\right)$ is holomorphic.

For any $\lambda \in \Omega_{\mathbf{i}}^{+}$, since $T$ is in $\mathcal{R} \mathcal{Q} \mathcal{B}_{n}(\Omega)$ together with and theorem $6.7, \lambda$ is a point of stability for $T_{c}-\lambda$ which means $T_{c}-\lambda$ is Fredholm and $\operatorname{dim}_{\mathbb{C}}\left(\operatorname{Ker}\left(T_{c}-\right.\right.$ $\omega)=n$ is constant on some neighborhood of $\lambda$. Thus, it follows from a result of Cowen and Douglas's paper (proposition 1.11 in [4]), that there exist $n$ analytic $\left(\mathcal{H}_{1} \oplus \mathcal{H}_{2}\right)$-valued functions $\left\{g_{1}(\lambda), \ldots, g_{n}(\lambda)\right\}$ as a basis of the space $\operatorname{Ker}\left(T_{c}-\lambda\right)$ for any $\lambda \in \Omega_{\mathbf{i}}^{+}$. According to the complex representation, proposition 2.3 and lemma 6.6, $\left\{g_{1}(\lambda), \ldots, g_{n}(\lambda)\right\}$ correspond to $n$ right analytic $\mathcal{H}_{q^{-} \text {-valued functions }}$ $\left\{f_{1}(\lambda), \ldots, f_{n}(\lambda)\right\}$ as a basis of the space $\operatorname{Ker}(T-\mathcal{I} \lambda)$ on $\mathbb{C}$. Therefore, $\left.f_{T}\right|_{\Omega_{\mathrm{i}}^{+}}$: $\Omega_{\mathbf{i}}^{+} \rightarrow \mathcal{G r}\left(n, \mathbb{C}, \mathcal{H}_{q}\right)$ is holomorphic.

Similar to a conclusion of Cowen and Douglas (corollary 1.13 in [4]), we have the following result.

Proposition 6.9. If $\Omega_{0}$ and $\Omega$ are two bounded connected open subsets of $\mathbb{H}$ with $\Omega_{0} \subseteq \Omega$, then $\mathcal{R} \mathcal{Q} \mathcal{B}_{n}(\Omega) \subseteq \mathcal{R} \mathcal{Q} \mathcal{B}_{n}\left(\Omega_{0}\right)$.

Proof. It is a straightforward corollary of theorem 6.8 and proposition 5.3.

Now let us show some concrete right quaternionic Cowen-Douglas operators.

Example 6.10. Suppose that $\left\{e_{i}\right\}_{i=1}^{\infty}$ is an ONB of $\mathcal{H}_{q}$. Let $M=\{z \in \mathbb{C} ;|z-i|<$ $1\}, \bar{M}=\{\bar{z} ; z \in M\}$ and $\widetilde{M}$ the axially symmetric completion of $M$. Consider

$$
T=\left[\begin{array}{ccccc}
\mathbf{i} & 1 & & & \\
& \mathbf{i} & 1 & & \\
& & \mathbf{i} & 1 & \\
& & & \ddots & \ddots
\end{array}\right] \begin{gathered}
e_{1} \\
e_{2} \\
e_{3} \\
\vdots
\end{gathered}
$$

Then

$$
T_{c}=\left[\begin{array}{cc}
A & 0 \\
0 & \bar{A}
\end{array}\right] \begin{aligned}
& \mathcal{H}_{1} \\
& \mathcal{H}_{2}
\end{aligned}
$$

where

$$
A=\left[\begin{array}{ccccc}
\mathbf{i} & 1 & & & \\
& \mathbf{i} & 1 & & \\
& & \mathbf{i} & 1 & \\
& & \ddots & \ddots
\end{array}\right] \begin{gathered}
e_{1} \\
e_{2} \\
e_{3} \\
\vdots
\end{gathered} \text { and } \bar{A}=\left[\begin{array}{ccccc}
-\mathbf{i} & 1 & & & \\
& -\mathbf{i} & 1 & & \\
& & -\mathbf{i} & 1 & \\
& & & \ddots & \ddots
\end{array}\right] \begin{gathered}
e_{1} \mathbf{j} \\
e_{2} \mathbf{j} \\
e_{3} \mathbf{j} \\
\vdots
\end{gathered}
$$

are complex linear operators on $\mathcal{H}_{1}=\bigvee_{\mathbb{C}}\left\{e_{i} ; i \in \mathbb{N}\right\}$ and $\mathcal{H}_{2}=\bigvee_{\mathbb{C}}\left\{e_{i} \mathbf{j} ; i \in \mathbb{N}\right\}$ respectively.

Notice that $A \in \mathcal{B}_{1}(M)$ and $\bar{A}-z$ is invertible for any $z \in M$ while $\bar{A} \in \mathcal{B}_{1}(\bar{M})$ and $A-\bar{z}$ is invertible for any $z \in M$. Then $T_{c}$ satisfies the conditions in theorem 
6.7 and hence $T \in \mathcal{R} \mathcal{Q B}_{1}(\widetilde{M})$. In fact, $\left(1, z-\mathbf{i}, \ldots,(z-\mathbf{i})^{n}, \ldots\right)$ is the restriction on $M$ of the homogeneous leaf-wise holomorphic curve on $\widetilde{M}$ induced by $T$.

Since the multiplication on quaternions is noncommutative, translation maybe change the indices of quaternionic Cowen-Douglas operators.

Example 6.11. Suppose that $\left\{e_{i}\right\}_{i=1}^{\infty}$ is an ONB of $\mathcal{H}_{q}$. Let $\Omega=\{z \in \mathbb{H} \backslash \mathbb{R} ;|z|<$ 1\}. Consider

$$
B=T-\mathbf{i} \mathcal{I}=\left[\begin{array}{ccccc}
0 & 1 & & & \\
& 0 & 1 & & \\
& & 0 & 1 & \\
& & \ddots & \ddots
\end{array}\right] \begin{gathered}
e_{1} \\
e_{2} \\
e_{3} \\
\vdots
\end{gathered}
$$

where $T$ is the operator defined in above example. Then

where

$$
B_{c}=\left[\begin{array}{cc}
B_{1} & 0 \\
0 & B_{2}
\end{array}\right] \mathcal{H}_{1},
$$

$$
B_{1}=\left[\begin{array}{ccccc}
0 & 1 & & & \\
& 0 & 1 & & \\
& & 0 & 1 & \\
& & & \ddots & \ddots
\end{array}\right] \begin{gathered}
e_{1} \\
e_{2} \\
e_{3} \\
\vdots
\end{gathered} \text { and } B_{2}=\left[\begin{array}{ccccc}
0 & 1 & & & \\
& 0 & 1 & & \\
& 0 & 1 & \\
& & \ddots & \ddots
\end{array}\right] \begin{gathered}
e_{1} \mathbf{j} \\
e_{2} \mathbf{j} \\
e_{3} \mathbf{j} \\
\vdots
\end{gathered}
$$

are complex linear operators on $\mathcal{H}_{1}=\bigvee_{\mathbb{C}}\left\{e_{i} ; i \in \mathbb{N}\right\}$ and $\mathcal{H}_{2}=\bigvee_{\mathbb{C}}\left\{e_{i} \mathbf{j} ; i \in \mathbb{N}\right\}$ respectively.

Notice that $B_{1}, B_{2} \in \mathcal{B}_{1}\left(\Omega_{\mathbf{i}}^{+}\right)$. Then $B_{c}$ satisfies the conditions in theorem 6.7 and hence $B \in \mathcal{R} \mathcal{Q} \mathcal{B}_{2}(\Omega)$. In fact, $f_{u}=\left(1, z, \ldots, z^{n}, \ldots\right)$ and $f_{d}=\left(\mathbf{j}, \mathbf{j} z, \ldots, \mathbf{j} z^{n}, \ldots\right)$. form a frame of the restriction on $\Omega_{\mathbf{i}}^{+}$of the homogeneous leaf-wise holomorphic curve on $\Omega$ induced by $B$.

It is obvious that the index of a quaternionic Cowen-Douglas operator is locally stable. However, it is not true in large scale.

Example 6.12. Suppose that $\left\{e_{i}\right\}_{i=1}^{\infty}$ is an ONB of $\mathcal{H}_{q}$. Let $M_{1}=\{z \in \mathbb{C} ; \mid z-$ $\frac{\mathbf{i}}{2}|<1| z+,\frac{\mathbf{i}}{2} \mid>1$ and $\left.\operatorname{Im}(z)>0\right\}$ and $M_{2}=\left\{z \in \mathbb{C} ;\left|z-\frac{\mathbf{i}}{2}\right|<1,\left|z+\frac{\mathbf{i}}{2}\right|<\right.$ 1 and $\operatorname{Im}(z)>0\}$, and let $\Omega_{1}$ and $\Omega_{2}$ be the axially symmetric completion of $M_{1}$ and $M_{2}$ respectively. Consider

$$
B=\left[\begin{array}{ccccc}
\frac{\mathbf{i}}{2} & 1 & & & \\
& \frac{\mathbf{i}}{2} & 1 & & \\
& & \frac{\mathbf{i}}{2} & 1 & \\
& & & \ddots & \ddots
\end{array}\right] \begin{gathered}
e_{1} \\
e_{2} \\
e_{3} \\
\vdots
\end{gathered} .
$$

We will show $B \in \mathcal{R} \mathcal{Q} \mathcal{B}_{1}\left(\Omega_{1}\right)$ and $B \in \mathcal{R} \mathcal{Q} \mathcal{B}_{2}\left(\Omega_{2}\right)$.

It is a routine to check conditions $(a-c)$ in definition 6.1. So we need only consider $(d)$.

The complex representation of $B$ is

$$
B_{c}=\left[\begin{array}{cc}
B_{1} & 0 \\
0 & B_{2}
\end{array}\right] \mathcal{H}_{1}
$$


where

$$
B_{1}=\left[\begin{array}{ccccc}
\frac{\mathbf{i}}{2} & 1 & & & \\
& \frac{\mathbf{i}}{2} & 1 & & \\
& \frac{\mathbf{i}}{2} & 1 & \\
& & & \ddots & \ddots
\end{array}\right] \begin{gathered}
e_{1} \\
e_{2} \\
e_{3}
\end{gathered} \text { and } B_{2}=\left[\begin{array}{cccccc}
-\frac{\mathbf{i}}{2} & 1 & & & \\
& -\frac{\mathbf{i}}{2} & 1 & & \\
& & -\frac{\mathbf{i}}{2} & 1 & \\
& & & \ddots & \ddots
\end{array}\right] \begin{gathered}
e_{1} \mathbf{j} \\
e_{2} \mathbf{j} \\
e_{3} \mathbf{j} \\
\vdots
\end{gathered}
$$

are complex linear operators on $\mathcal{H}_{1}=\bigvee_{\mathbb{C}}\left\{e_{i} ; i \in \mathbb{N}\right\}$ and $\mathcal{H}_{2}=\bigvee_{\mathbb{C}}\left\{e_{i} \mathbf{j} ; i \in \mathbb{N}\right\}$ respectively.

Notice that $B_{1} \in \mathcal{B}_{1}\left(M_{1}\right)$ and $B_{2}-z$ is invertible for any $z \in M_{1}$ while $B_{2} \in \mathcal{B}_{1}\left(\overline{M_{1}}\right)$ and $B_{1}-\bar{z}$ is invertible for any $z \in M_{1}$. Then $B \in \mathcal{R} \mathcal{Q} \mathcal{B}_{1}\left(\Omega_{1}\right)$. On the other hand, since $B_{1}, B_{2} \in \mathcal{B}_{1}\left(M_{2}\right)$, we have $B \in \mathcal{R} \mathcal{Q B}_{2}\left(\Omega_{2}\right)$.

Now let us consider the classification of $\mathcal{R} \mathcal{Q} \mathcal{B}_{n}(\Omega)$ in the sense of quaternion unitary equivalence. Denote by $s_{T}$ the QEHM on $\left(\Omega_{\mathbf{i}}^{+} \times \mathbb{C}^{n}, \Omega_{\mathbf{i}}^{+}, \pi\right)$ induced by $f_{T}$. Then we have the main result.

Theorem 6.13. Let $T, S \in \mathcal{R} \mathcal{Q} \mathcal{B}_{n}(\Omega)$. Then the following statements are equivalent:

(a) $T$ and $S$ are quaternion unitarily equivalent in $\mathcal{L}\left(\mathcal{H}_{q}\right)$;

(b) The two n-dimensional homogeneous leaf-wise holomorphic curves $f_{T}$ and $f_{S}$ are congruent.

(c) The two $n$-dimensional QEHBs $\left(\Omega_{\mathbf{i}}^{+} \times \mathbb{C}^{n}, \Omega_{\mathbf{i}}^{+}, \pi, s_{T}\right)$ and $\left(\Omega_{\mathbf{i}}^{+} \times \mathbb{C}^{n}, \Omega_{\mathbf{i}}^{+}, \pi, s_{S}\right)$ are holomorphic q-isomorphic.

Proof. According to theorem 5.5, $(b)$ and $(c)$ are equivalent. It suffices to show that $(a)$ and $(b)$ are equivalent.

$(a) \Longrightarrow(b)$. If $U$ is the quaternion unitary operator such that $U T=S U$, then $U(\operatorname{Ker}(T-\mathcal{I} \omega))=\operatorname{Ker}(S-\mathcal{I} \omega)$. Hence $f_{T}$ and $f_{S}$ are congruent.

$(b) \Longrightarrow(a)$. If $U$ is the quaternion unitary operator such that $U f_{T}(\omega)=f_{S}(\omega)$, then for any $x \in \operatorname{Ker}(T-\mathcal{I} \omega)$,

$$
U T(x)=U(x \omega)=(U x) \omega=S(U x)=S U(x) .
$$

Since

$$
\overline{\dot{+}_{\omega \in \Omega} \operatorname{Ker}(T-\mathcal{I} \omega)}=\mathcal{H}_{q},
$$

we have $U T=S U$.

At last, we will investigate that quaternion unitary equivalence for quaternionic operators is strictly stronger than complex unitary equivalence for their complex representations.

Proposition 6.14. Let $F, G \in \mathcal{L}\left(\mathcal{H}_{q}\right)$. If $F$ and $G$ are quaternion unitarily equivalent, then their complex representations $F_{c}$ and $G_{c}$ are complex unitarily equivalent.

Proof. Notice that if $U$ is a quaternion unitary operator on $\mathcal{H}_{q}$, then its complex representations $U_{c}$ is a complex unitary operator. Then the result is obtained immediately.

However, the inverse is not true. In particular, we have counterexamples in quaternionic Cowen-Douglas operators. 
Example 6.15. Suppose that $\left\{e_{i}\right\}_{i=1}^{\infty}$ is an ONB of $\mathcal{H}_{q}$. Let $M=\{z \in \mathbb{C} ;|z-i|<$ $1\}$ and $\Omega$ the axially symmetric completion of $M$. Denote

$$
F=\left[\begin{array}{ccccc}
\mathbf{j} & 1 & & & \\
& \mathbf{i} & 1 & & \\
& \mathbf{i} & 1 & \\
& & \ddots & \ddots
\end{array}\right] \begin{array}{ccccc}
e_{1} & \\
e_{2} \\
e_{3}
\end{array} \text { and } \quad G=\left[\begin{array}{ccccc}
\mathbf{k} & 1 & & & \\
& \mathbf{i} & 1 & & \\
& \mathbf{i} & 1 & \\
& & \ddots & \ddots
\end{array}\right]
$$

Then $F, G \in \mathcal{R} \mathcal{Q} \mathcal{B}_{1}(\Omega)$ and they are not quaternion unitarily equivalent while their complex representations are complex unitarily equivalent.

Proof. Write

$$
F=T+K
$$

where

$$
T=\left[\begin{array}{ccccc}
\mathbf{i} & 1 & & & \\
& \mathbf{i} & 1 & & \\
& & \mathbf{i} & 1 & \\
& & & \ddots & \ddots
\end{array}\right] \text { and } K=\left[\begin{array}{cccc}
\mathbf{j}-\mathbf{i} & 0 & 0 & \cdots \\
0 & 0 & 0 & \cdots \\
0 & 0 & 0 & \cdots \\
\vdots & \vdots & \vdots & \ddots
\end{array}\right]
$$

Then $T \in \mathcal{R} \mathcal{Q B}_{1}(\Omega)$ (see example 6.10) and $K$ is a compact operator. By theorem 6.7, $T_{c}$ is a Fredholm operator with index 1. Thus, $F_{c}=T_{c}+K_{c}$ is also a Fredholm operator with index 1 for $K_{c}$ is compact. To prove $F \in \mathcal{R} \mathcal{Q} \mathcal{B}_{1}(\Omega)$, it suffices to show that $\operatorname{dim}_{\mathbb{C}} \operatorname{Ker}\left(T_{c}-z\right)=1$ for $z \in M$ and $\bigvee_{\mathbb{C}}\left\{\operatorname{Ker}\left(T_{c}-z\right), \operatorname{Ker}\left(T_{c}-\right.\right.$ $\bar{z}) ; z \in M\}=\mathcal{H}_{1} \oplus \mathcal{H}_{2}$. Let $f_{1}(z)=z+\mathbf{j}$ and $f_{n}(z)=(z+\mathbf{i})(z-\mathbf{i})^{n-1}$ for $n \geq 2$. Put $f(z)=\left(f_{1}(z), f_{2}(z), \ldots\right)$ under the ONB $\left\{e_{i}\right\}_{i=1}^{\infty}$. Then we can see $\operatorname{Ker}\left(T_{c}-z\right)=\{\lambda f(z) ; \lambda \in \mathbb{C}\}$ for $z \in M$. Moreover, $f(z)$ is the homogeneous leaf-wise holomorphic curve induced by $F$. Since

$$
\bigvee_{\mathbb{H}}\left\{f^{(j)}(\mathbf{i}) ; j=0,1, \ldots\right\}=\mathcal{H}_{q}
$$

it follows from proposition 5.4 and theorem 6.7 that

$$
\bigvee_{\mathbb{C}}\left\{\operatorname{Ker}\left(T_{c}-z\right), \operatorname{Ker}\left(T_{c}-\bar{z}\right) ; z \in M\right\}=\mathcal{H}_{1} \oplus \mathcal{H}_{2}
$$

Similarly, $G \in \mathcal{R} \mathcal{Q} \mathcal{B}_{1}(\Omega)$ and $g(z)=\left(g_{1}(z), g_{2}(z), \ldots\right)$ is the homogeneous leaf-wise holomorphic curve induced by $F$, where $g_{1}(z)=z+\mathbf{k}$ and $g_{n}(z)=$ $(z+\mathbf{i})(z-\mathbf{i})^{n-1}$ for $n \geq 2$.

Now, one can see that $F$ and $G$ are not quaternion unitarily equivalent. By theorem 6.13, if $F$ and $G$ are quaternion unitarily equivalent, then $f(z)$ and $g(z)$ are congruent. Consequently, there exists a complex holomorphic function $h$ on $\Omega_{\mathbf{i}}^{+}$such that $f(z) \cdot h(z)=g(z)$ for all $z \in \Omega_{\mathbf{i}}^{+}$. However, $f_{1}(z)=z+\mathbf{j}$ multiplied by any complex number can not be $g_{1}(z)=z+\mathbf{k}$ which contradicts with the assumption. In other words, the two QEHBs $\left(\Omega_{\mathbf{i}}^{+} \times \mathbb{C}, \Omega_{\mathbf{i}}^{+}, \pi, s_{F}\right)$ and $\left(\Omega_{\mathbf{i}}^{+} \times \mathbb{C}, \Omega_{\mathbf{i}}^{+}, \pi, s_{G}\right)$ are not holomorphic q-isomorphic.

On the other hand, it is obvious that the complex restrictions of $\left(\Omega_{\mathbf{i}}^{+} \times \mathbb{C}, \Omega_{\mathbf{i}}^{+}, \pi, s_{F}\right)$ and $\left(\Omega_{\mathbf{i}}^{+} \times \mathbb{C}, \Omega_{\mathbf{i}}^{+}, \pi, s_{G}\right)$ are the same. Moreover, we will show that the complex 
representations of $F$ and $G$ are complex unitarily equivalent. Notice that

$$
F_{c}=\left[\begin{array}{cc}
A & -\bar{B} \\
B & \bar{A}
\end{array}\right] \begin{gathered}
\mathcal{H}_{1} \\
\mathcal{H}_{2}
\end{gathered} \quad \text { and } \quad G_{c}=\left[\begin{array}{cc}
A & -\bar{C} \\
C & \bar{A}
\end{array}\right] \mathcal{H}_{1}, \mathcal{H}_{2},
$$

where

$$
A=\left[\begin{array}{ccccc}
0 & 1 & & & \\
& \mathbf{i} & 1 & & \\
& & \mathbf{i} & 1 & \\
& & & \ddots & \ddots
\end{array}\right], B=\left[\begin{array}{cccc}
1 & & & \\
& 0 & & \\
& & 0 & \\
& & & \ddots
\end{array}\right] \text { and } C=\left[\begin{array}{cccc}
-\mathbf{i} & & & \\
& 0 & & \\
& & 0 & \\
& & & \ddots
\end{array}\right] \text {. }
$$

Consider

$$
V=\left[\begin{array}{cc}
I d & 0 \\
0 & -\mathbf{i} I d
\end{array}\right] \begin{aligned}
& \mathcal{H}_{1} \\
& \mathcal{H}_{2}
\end{aligned}
$$

where $I d$ means the identity operator. Then $V$ is a complex unitary operator (notice that $V$ can not be a complex representation of a quaternion unitary operator) and $V F=G V$.

Acknowledgement. This work was supported by the National Nature Science Foundation of China (Grant No. 11001099).

\section{REFERENCES}

1. S.L. Adler, Quaternionic Quantum Mechanics and Quantum Fields, Oxford U.P., New York, 1994.

2. F. Colombo, G. Gentili, I. Sabadini and D. Struppa, Extension results for slice regular functions of a quaternionic variable, Adv. Math. 222 (2009), no. 5, 1793-1808.

3. F. Colombo, I. Sabadini and D. Struppa, Noncommutative Functional Calculus: Theory and Applications of Slice Hyperholomorphic Functions, Progress in Mathematics, Vol. 289, Springer Basel AG, Basel, 2011.

4. M.J. Cowen and R.G. Douglas, Complex geometry and operator theory, Acta Math. 141 (1978), no. 1, 187-261.

5. S. De Leo and G. Scolarici, Right eigenvalue equation in quaternionic quantum mechanics, J. Phys. A: Math. Gen. 33 (2000), no. 15, 2971-2995.

6. S. De Leo, G. Scolarici and L. Solombrino, Quaternionic eigenvalue problem, J. Math. Phys. 43 (2002), 5815-5829.

7. R. Feres and A. Zeghib, Leafwise holomorphic functions, Proc. Amer. Math. Soc. 131 (2003), 1717-1725.

8. D. Finkelstein, J.M. Jauch, S. Schiminovich and D. Speiser, Foundations of quaternion quantum mechanics, J. Math. Phys. 3 (1962), 207-220.

9. D. Finkelstein, J.M. Jauch and D. Speiser, Quaternionic representations of compact groups, J. Math. Phys. 4 (1963), 136-140.

10. C. Jiang and K. Ji, Similarity classification of holomorphic curves, Adv. Math. 215 (2007), 446-468.

11. S. Natarajan and K. Viswanath, Quaternionic representations of compact metric groups, J. Math. Phys. 8 (1967), 582-589.

12. C.S. Sharma and T.J. Coulson, Spectral theory for unitary operators on a quaternionic Hilbert space, J. Math. Phys. 28 (1987), no. 9, 1941-1946.

13. K. Viswanath, Normal Operators on Quaternionic Hilbert Spaces, Trans. Amer. Math. Soc. 162 (1971), 337-350.

14. F. Zhang, Quaternions and Matrices of Quaternions, Linear Algebra Appl. 251 (1997), $21-57$. 
${ }^{1}$ School of Mathematics, Jilin university, 130012, Changchun, P.R.China.

E-mail address: houbz@jlu.edu.cn

2 Department of Mathematics, Liaoning University, 110036, Shenyang, P. R. ChInA.

E-mail address: tiangeng09@mails.jlu.edu.cn 